\title{
A New Wavelets Image Fusion Strategy
}

\author{
M. Santos, G. Pajares, M. Portela, J.M de la Cruz \\ Dpto. Arquitectura de Computadores y Automática. Facultad de CC. Físicas. \\ Universidad Complutense de Madrid. 28040-Madrid, Spain \\ msantos@dacya.ucm.es
}

\begin{abstract}
The aim of image fusion is to combine information from multiple images of the same scene. The result of image fusion is a new image, which is more suitable for human and machine perception or further image-processing tasks such as segmentation, feature extraction and object recognition. Different fusion methods have been proposed in the literature. This paper presents new methods based on the computation of local and global gradient. A comparative analysis is carried out against other existing strategies. The results are encouraging.
\end{abstract}

\section{Introduction}

Pixel-level fusion serves to increase the useful information content of an image so that the performance of image-processing tasks such as segmentation, feature extraction and object recognition can be improved $[1,2]$. This paper presents a new pixel-level wavelet fusion approach based on the gradient concept. The gradient concept exploits the relevant information derived from the grey level variation in the images, particularly around the edges. Initially for each pixel location a gradient matrix is computed. Then taking into account neighbourhood gradient interactions through non-overlapping or overlapping areas, such matrix is modified by a relaxation procedure.

Therefore, we propose a new strategy based on the gradient, where two processes are involved: local and global. The use of the local and global gradient approaches in image fusion makes up the main findings of this paper. As our method is based on the gradient, it is suitable for images where edges are abundant, such as indoor environments.

This paper is organised as follows. Section 2 presents an overview about wavelet decomposition and fusion merging methods. In section 3 we propose new merging methods based on the gradient computation. Section 4 presents some experimental results that have been satisfactory compared to other classical existing methods. Finally section 5 summarizes the conclusions. 


\section{Overview: Wavelet Decomposition and Merging Fusion Methods}

Many researchers recognised that multi-scale transforms are very useful for image fusion $[3,4,5]$. Nowadays the wavelet mathematical theory has received special attention for multi-scale representations. This is because using Discrete Wavelets Transform (DWT) it is possible to reach the desired decomposition level preserving the image information [2].

In order to achieve the fused image (i.e. the improved image), a sequence of operations is required. Figure 1 summarises them as a block diagram. Two original input images are to be fused. The DWT is applied to each one, and the result of this transformation is a Multi-scale image at different resolution levels. As we will see in the next section, this transformation produces the called frequency bands. From the multi-scale images a Select and Merging $(S \& M)$ method is applied to achieve a Fused Multi-scale image. Then, by applying the Inverse Discrete Wavelets Transform (IDWT) the final fused image is obtained, which contains the best information from the two incoming images. This paper is only concerned with the $S \& M$ strategy. The DWT and IDWT operations are called analysis and synthesis respectively.

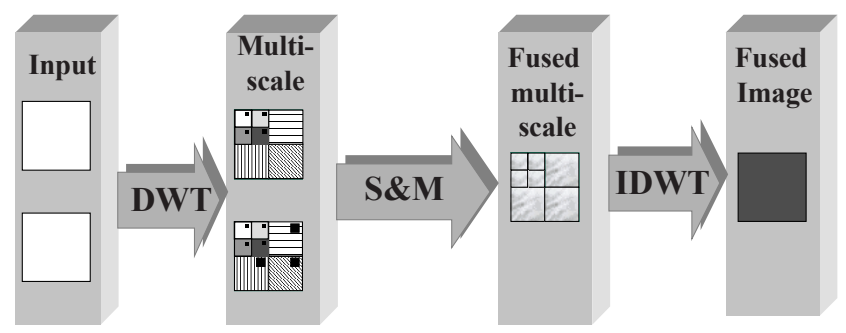

Fig. 1. Fusion Scheme

\subsection{Discrete Wavelet Transform (DWT)}

According to $[6,7,8,9,10]$, the 2-D wavelet analysis operation consists in filtering and down-sampling horizontally using the 1-D lowpass filter $L$ and the highpass filter $H$ to each row in the image $I(x, y)$, producing the coefficient matrices $I_{L}(x, y)$ and $I_{H}(x, y)$. In this paper, we have used the Haar filters. Vertically filtering and downsampling follows, using the lowpass and highpass filters $L$ and $H$ to each column in $I_{L}(x, y)$ and $I_{H}(x, y)$, and produces four subimages $I_{L L}(x, y), I_{L H}(x, y), I_{H L}(x, y)$ and $I_{H H}(x, y)$ for one level of decomposition. $I_{L L}(x, y)$ is a smooth subimage corresponding to the low frequency band of the multi-scale decomposition and can be considered as a smoothed and subsampled version of the original image $I(x, y)$, i.e. it represents the coarse approximation of $I(x, y) . I_{L H}(x, y), I_{H L}(x, y)$ and $I_{H H}(x, y)$ are detail subimages, which represent the horizontal, vertical and diagonal directions of the image $I(x, y)$. They are respectively low-high, high-low and high-high frequency bands. Details about the implementation of the DWT and IDWT can be found in $[6,11]$. Figure 2 shows a representation of a two level image decomposition. We can notice that there is only one low frequency band, $I_{L L I}$ in the two-level decomposition. The rest of bands 
are high-frequency bands. This can be extended to any K-level where only a low frequency band is available, $I_{L L(K-1)}$.

\begin{tabular}{|l|l|l|}
\hline$I_{L L I}$ & $I_{L H I}$ & \multirow{2}{*}{$I_{L H}$} \\
\cline { 1 - 2 }$I_{H L I}$ & $I_{H H I}$ & \\
\hline \multicolumn{1}{|l|}{$I_{H L}$} & & $I_{H H}$ \\
\hline
\end{tabular}
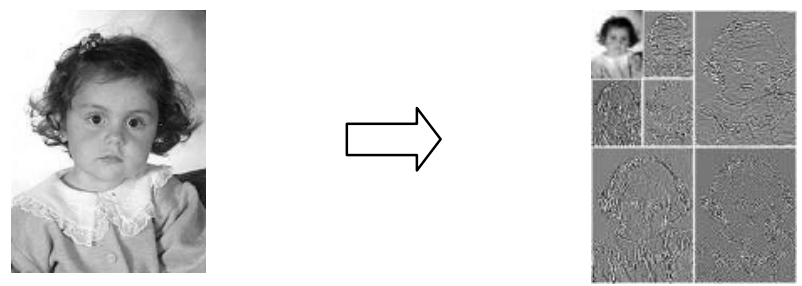

Fig. 2. A representation of a two-level image decomposition and an example

\subsection{Select and Merging Strategy (S\&M)}

In figure 1, the Multi-scale block shows the 2-D structures of a multi-scale transform with two decomposition levels. A given textured square corresponds to the same decomposition level in the two images. The black small squares in different frequency bands correspond to the same group of pixels, which indicate the spatial localisation of the transform. These are the pixels to be fused. Hence, only the fusion at the same resolution level is allowed through the called fusion rule [1].

Generally an image $I$ has its multi-scale decomposition representation denoted as $D_{I}$. Given two inputs images $X$ and $Y$, a fusion rule can be defined in order to obtain the resulting combination $Z$. Consider the following example, assume that images $X$ and $Y$ have been two-level decomposed, this means that we can fuse for example $X_{L L 1}$ and $Y_{L L 1}$ obtaining $Z_{L L 1}$. They should be represented as $D_{L L k}^{X}, D_{L L k}^{Y}$ and $D_{L L k}^{Z}$ respectively. With simplification purposes, for the first decomposition level, i.e. $k=0$, we do not use the sub-index $k$. Some merging strategies can be found in [1], such as:

$$
\text { Maximum: } D_{i j k}^{Z}(p)=\max \left(\| D_{i j k}^{X}(p)|,| D_{i j k}^{Y}(p) \mid\right),
$$

Arithmetic average combination $D_{i j k}^{Z}(p)=w_{X}(p) D_{i j k}^{X}(p)+w_{Y}(p) D_{i j k}^{Y}(p)$

$$
\text { Geometric average combination } D_{i j k}^{Z}(p)=\sqrt{D_{i j k}^{X}(p) D_{i j k}^{Y}(p)}+b
$$

where $w_{X}, w_{Y}$ are weighting coefficients, $p$ denotes the pixel position and $b$ is an arbitrary constant. The fact that the above magnitudes are functions of $p$ means that they can vary according to the pixel position.

In the next section, a new fusion merging strategy based on the gradient is introduced, which takes into account grey level differences at each position $p$. Also the position concept $p$ is extended, so that it includes $p$ and an area surrounding $p$. Different approaches are analysed and studied. 


\section{Gradient Merging Based Methods}

This section analyses new strategies based on the gradient concept at each location $p$, which is computed considering a region $R$ surrounding $p$ as follows,

$$
G_{i j k}^{h}(p)=\sum_{\substack{q \in A \\ q \neq p}}\left(D_{i j k}^{h}(q)-D_{i j k}^{h}(p)\right)
$$

where $i j$ represents a frequency band $L L, L H, H L$ or $H H ; k$ is the decomposition level; $h$ is a given input image $X$ or $Y ; A$ is the area surrounding $p$ and $q$ is a location in $A$.

From fig. 1 and equation (1), the following algorithm LGM (Local Gradient Method) performs the fusion process:

1. Initialisation: define the area size $A$ which will be used around each location $p$ and the decomposition level $K$. Set $k=-1$.

2. Inputs: images $X$ and $Y$; set $U=X$ and $V=Y$

3. Multi-scale wavelets decomposition: obtain the transformed subimages.

$$
k=k+1 ; U, V \rightarrow \mathrm{DWT} \rightarrow D_{L L k}^{U, V}, D_{L H k}^{U, V}, D_{H L k}^{U, V}, D_{H H k}^{U, V}
$$

4. Fused image: Select: for each location $p$ at $(x, y)$ in the transformed subimages compute the following gradient values using equation (1):

$$
G_{i j k}^{U}(p) ; G_{i j k}^{V}(p) \text { with ij = LL, LH, HL and HH }
$$

Merge: for each location $p$ compute the following values

$$
\begin{aligned}
& g_{i j k}(p)=\max \left\{G_{i j k}^{U}(p)|,| G_{i j k}^{V}(p) \mid\right\} \text { with ij }=\text { LL, LH, HL and HH } \\
& D_{i j k}^{W}(p)=D_{i j k}^{U}(p) \text { if } g_{i j k}(p) \text { comes from U else } D_{i j k}^{W}(p)=D_{i j k}^{V}(p)
\end{aligned}
$$

5. Check for the end of the multi-scale decomposition:

if $k<K$ then set $U=D_{L L k}^{U}, V=D_{L L k}^{V}$ and go to 3; else go to 6

6. While $\mathrm{k} \neq-1$

apply IDWT to $D_{L L k}^{W}, D_{L H k}^{W}, D_{H L k}^{W}$ and $D_{H H k}^{W}$ obtaining $D_{L L(k-1)}^{W} ; \mathrm{k}=\mathrm{k}-1$

7. Output: fused image $\mathrm{Z}=D_{L L k}^{W}$

In this paper $k=0$, i.e., a unique decomposition level is used without lack of generalisation.

We have carried out our experiments with the two images in Figure 3. They are multifocus images where depending on the image the same area appears defocused or focused. The goal is to choose the best area from each image, so that the resulting 
image is a perfect focused image. Our gradient method is motivated by the fact that the blurred effect is more evident around the edges and the degree of blurring can be measured by the gradient.
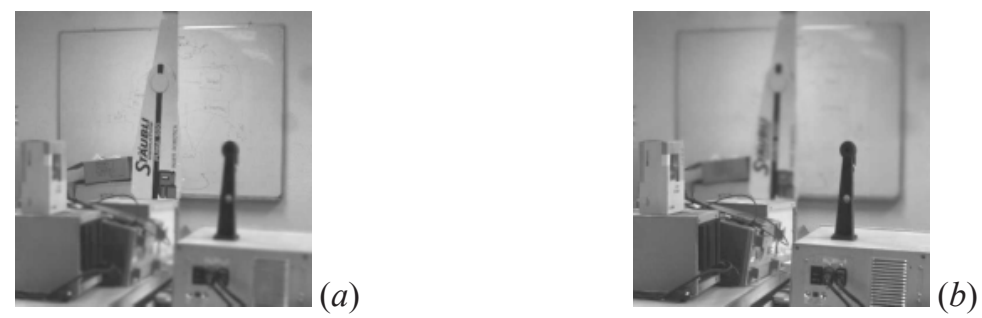

Fig. 3. Input images: (a) image 1 (focus on far); (b) image 2 (focus on close)

We have verified that the results obtained with the above algorithm LGM, when applying the local gradient straightforward, are satisfactory if comparing them to other methods, although they can be improved. With such purpose we have studied and analysed different approaches derived from this algorithm. As expected, some mistakes appear around the edges. Hence, we have focused on the behaviour of our method near the edges. Within the different methods we have implemented, the following three alternatives have been analysed in deep: 1) Local gradient applied to the $I_{H H}$ subimage; 2) Local gradient applied to a region, and 3) Global gradient.

\subsection{Local Gradient Applied to the $\mathrm{I}_{\mathrm{HH}}$ Subimage}

In the step 4 of our algorithm, the different $D_{i j k}^{W}(p)$ are obtained for the four subimages. This could result in, for example, two values are selected from image $X$ and the other two from image $Y$. To avoid this, the four coefficients are selected from the same image by choosing the most significant subimage, the $I_{H H}$ which contains high frequency structures. The original algorithm should be modified in step 4 as follows:

Compute $g_{i j k}(p)=\max \left\{G_{i j k}^{U}(p)|,| G_{i j k}^{V}(p) \mid\right\}$ with $\mathrm{ij}=\mathrm{HH}$

If the maximum $g_{H H k}(p)$ value comes from $U$, then $D_{H H k}^{W}(p)=G_{H H k}^{U}(p)$ and the remainder $D_{i j k}^{W}(p)$ with $i j=L L, H L$ and $L H$ are also selected from $U$, otherwise from $V$. This step is repeated for each $p$ of the $\mathrm{HH}$ subimages coming from $\mathrm{X}$ and $\mathrm{Y}$.

\subsection{Local Gradient Applied to a Region}

Instead of using the gradient at each location $p$ we consider a region around $p$. That allows considering more information. We compute the gradient average value in a region and use the following criterion, which alters the step 4 of the algorithm as follows. Compute (2), 


$$
g_{i j k}(p)=\max \left\{\left|\frac{1}{n_{R}} \sum_{q_{h} \in \mathfrak{R}} G_{i j k}^{U}\left(q_{h}\right)\right|,\left|\frac{1}{n_{R}} \sum_{q_{h} \in \Re} G_{i j k}^{V}\left(q_{h}\right)\right|\right\}
$$

where $\mathfrak{R}$ is a rectangular region of size $n \times m, q_{h}$ are the coefficients belonging to $\mathfrak{R}$ and $n_{R}=n \times m$ is the number of coefficients in $\mathfrak{R}$. Select $\mathrm{W}=\mathrm{U}$ in $D_{i j k}^{W}(p)$ if $g_{i j k}(p)$ comes from $\mathrm{U}$, otherwise $\mathrm{W}=\mathrm{V}$. The size of the region is defined by the user. In our approach the region has size $3 \times 3$ around $p$, hence $n=9$. As before, if the maximum value comes from $U$, then the remainder $D_{i j k}^{W}(p)$ with $i j=L L, L H$ and $H L$ are selected from $U$, otherwise from $V$.

\subsection{Global Gradient}

\subsubsection{Version 1}

Instead of using only the $I_{H H k}$ subimage, we have used the three subimages that contain some high-pass filtering, namely: $I_{L H k}, I_{H L k}$ and $I_{H H k}$. Now the step 4 is,

$$
g_{i j k}(p)=\max \left\{\left|\sum_{i j=S} \frac{1}{n_{R}} \sum_{q_{h} \in \Re} G_{i j k}^{U}\left(q_{h}\right)\right|,\left|\sum_{i j=S} \frac{1}{n_{R}} \sum_{q_{h} \in \mathcal{K}} G_{i j k}^{V}\left(q_{h}\right)\right|\right\}
$$

where $S=\{L H, H L, H H\}$ and $i j=L L, L H, H L$ and $H H$. If the maximum $g_{i j k}(p)$ value comes from $U$, then $D_{i j k}^{W}(p)=G_{i j k}^{U}(p)$, otherwise $\mathrm{W}=V$.

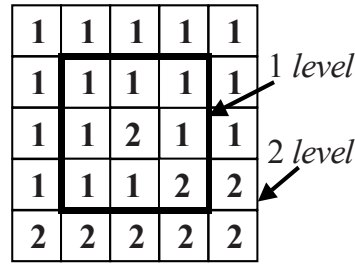

(a)
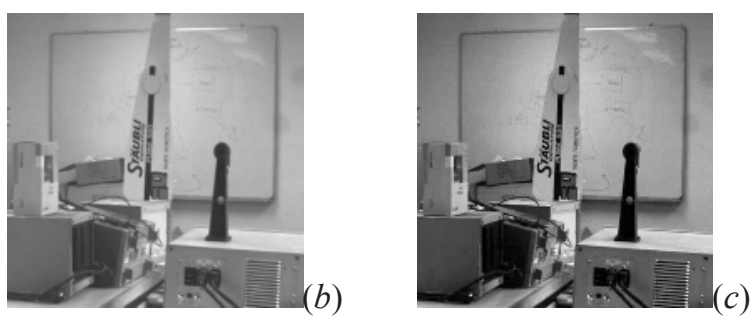

Fig. 4. Fused image obtained through global gradient (Version1)

So, each of the regions of the fused image is taken from one of the input images following that criterion, as it is represented in Figure 8. Assume $N \times M$ the $U$ and $V$ sizes, we build that global matrix of size $\frac{N}{n} \times \frac{M}{m}$ in which each element is labelled as "1" or "2" depending on if the selection comes from $U$ or $V$ respectively. That matrix is called the global matrix. Now we start a relaxation process to modify the global matrix values. We define a region and its labels are modified according to a majority criterion. In this way, for the example shown in figure $4(a)$, the central element, labelled with 2 , can be expected to be a mistake, as it is surrounded by regions that will be taken from the other input image. So, it would be exchanged for " 1 ". This 
process continues until no modifications appear. After this, we select the $D_{i j k}^{W}(p)$ coefficients according to the final labels in the global matrix.

The number of elements of the global matrix under consideration can be increased, to apply the relaxation process taking into account more information. For example, a second level can be considered (Figure $4 a$ ), and that could vary the final results.

\subsubsection{Version 2}

We compute the eight values of the gradient surrounding the central one at position p:

$$
g_{i j}^{T}=\left|\sum_{q_{h} \in \Re} G_{i j k}^{T}\left(q_{h}\right)\right| \text { for } \mathrm{ij}=\mathrm{LL}, \mathrm{LH}, \mathrm{HL} \text { and } \mathrm{HH} \text {; and } \mathrm{T}=\mathrm{U}, \mathrm{V} .
$$

If the majority of maximum $g_{i j}$ values come from $\mathrm{U}$, the corresponding global matrix element is labelled as "1" otherwise as "2". The relaxation process is executed as in version 1 .

\subsubsection{Version 3}

In this version, the global matrix is built as in version 1, but we introduce an additional constraint in its updating process. Indeed, a label "1" in the global matrix is changed if most of the labels in its neighbourhood are "2" as in version 1, and also if $d_{U}>d_{V}$, new in version 3, equation (4). And vice versa for labels "2".

$$
d_{T}=\left|\sum_{i j=S} \sum_{q_{h} \in \Re} D_{i j k}^{T}\left(q_{h}\right)\right| \quad \text { where } T=\{U, V\}
$$

That refers to the fact that the element which has the highest absolute value of the gradient has more information about the details of the image.

\subsubsection{Version 4}

Until now, we have split the input images into non-overlapping regions. This version considers four overlapping areas surrounding the central region.

\section{Experimental Results}

In order to verify the performance of the proposed local and global gradient methods, we compare different merging methods including classical ones: Maximum (MAX); Arithmetic (AAV) and Geometric average (GAV); Local Gradient Method (LGM), Local gradient applied to the $I_{H H}$ (LGI) and to a region (LGR); Global Gradient Version 1 (GG1), Version 2 (GG2), Version 3 (GG3) and Version 4 (GG4). As a quality measure we use an objective fidelity criterion: the root-mean-square error $e_{r m s}$. This comparison is carried out against the benchmark image in figure $4(b)$. 
Table 1. Root-mean-squared error for different methods

$\begin{array}{lcccccccccc}\text { Methods } & \boldsymbol{M A} \boldsymbol{A} & \boldsymbol{A} \boldsymbol{A} \boldsymbol{V} & \boldsymbol{G A \boldsymbol { V }} & \boldsymbol{L G M} & \boldsymbol{L G I} & \boldsymbol{L G} \boldsymbol{R} & \boldsymbol{G G} \boldsymbol{1} & \boldsymbol{G G} \boldsymbol{2} & \boldsymbol{G G 3} & \boldsymbol{G G} \boldsymbol{4} \\ \mathrm{e}_{\mathrm{rms}} & 4.6012 & 5.0870 & 7.3385 & 2.5656 & 2.1943 & 1.7196 & 1.3555 & 1.6222 & 1.4525 & 1.3767\end{array}$

From the results in table 1:1) As expected, the best results are achieved with our proposed gradient global strategies, as a relaxation process always improves the local results; 2) The best performance is achieved with version 1 (Figure 4c). This is because such method uses all subimages containing high frequency information. Therefore, we can point out that the errors come from the edges and it is embedded in the gradient. This assertion can be verified also with the results obtained in version 3 which are slightly worst than those obtained in version 1, i.e. additional information out of the gradient does not improve the results. The use of overlapping regions (version 4) improves the results of version 3, where non-overlapping regions are used.

\section{Conclusion}

We have developed several gradient based methods with fusion merging purposes. We have found its performance against other methods, with encouraging results. We have verified that the best performance is achieved applying gradient based strategies, particularly with the global ones. This means that the gradient appears as a good strategy in image fusion applications using wavelets.

\section{References}

[1] Z. Zhang and R.S. Blum, A categorization of Multiscale-Decomposition-Based Image Fusion Schemes with a Performance Study for a Digital Camera Application, Proceedings of the IEEE 87(8) (1999) 1315-1326.

[2] H. Li, B.S. Manjunath, S.K. Mitra, Multisensor Image Fusion Using the Wavelet Transform, Graphical Models and Image Processing 57(3) (1995) 235245.

[3] P.J. Burt, E. Adelson, The Laplacian pyramid as a compact image code, IEEE Trans. Commun. 31 (1983) 532-540.

[4] E. H. Adelson, C.H. Anderson, J.R. Bergen, P.J. Burt, J. Ogden, Pyramid methods in image processing, RCA Engineer, 29(6) (1984) 33-41.

[5] T. Lindeberg, Scale-Space Theory in Computer Vision, Kluwer, Norwell: MA, 1994.

[6] S. Mallat, A Wavelet Tour of Signal Processing, Academic Press, $2^{\text {nd }}$ ed, 1999.

[7] E.J. Stollnitz, T.D. DeRose, D.H. Salesin, Wavelets for Computer Graphics: A Primer, Part 1, IEEE Computer Graphics and Applications 15(3) (1995) 76-84.

[8] I. Daubechies, Ten Lectures on Wavelets, SIAM, Philadelphia, 1992.

[9] I. Daubechies, Orthonormal Bases of Compactly Supported Wavelets, Comm. Pure Applied Mathematics 41 (1988) 909-996.

[10] J.L. Starck, F. Murtagh, A. Bijaoui, Image Processing and Data Analysis: The multiscale approach. Cambridge, University Press, 2000.

[11] G. Pajares, J. M. de la Cruz, Visión por Computador: Imágenes Digitales y Aplicaciones, RA-MA: Madrid, 2001. 